Chapter 8

\title{
Discrete Barrier and Lookback Options
}

\author{
S.G. Kou
}

Department of Industrial Engineering and Operations Research, Columbia University

E-mail: sk75@columbia.edu

\begin{abstract}
Discrete barrier and lookback options are among the most popular path-dependent options in markets. However, due to the discrete monitoring policy almost no analytical solutions are available for them. We shall focus on the following methods for discrete barrier and lookback option prices: (1) Broadie-Yamamoto method based on fast Gaussian transforms. (2) Feng-Linetsky method based on Hilbert transforms. (3) A continuity correction approximation. (4) Howison-Steinberg approximation based on the perturbation method. (5) A Laplace inversion method based on Spitzer's identity. This survey also contains a new (more direct) derivation of a constant related to the continuity correction approximation.
\end{abstract}

\section{Introduction}

Discrete path-dependent options are the options whose payoffs are determined by underlying prices at a finite set of times, whereas the payoff of a continuous path-dependent option depends on the underlying price throughout the life of the option. Due to regulatory and practical issues, most of path-dependent options traded in markets are discrete path-dependent options.

Among the most popular discrete path-dependent options are discrete Asian options or average options, discrete American options or Bermuda options, discrete lookback options and discrete barrier options. The payoff of a discrete Asian option depends on a discrete average of the asset price. For example, a standard discrete (arithmetic European) Asian call option has a payoff $\left(\frac{1}{n} \sum_{i=1}^{n} S\left(t_{i}\right)-K\right)^{+}$at maturity $T=t_{n}$, where $t_{1}, t_{2}, \ldots, t_{n}$ are monitoring points, $K$ is the strike price of the call option, and $S(t)$ is the asset price at time $t$; see Zhang (1998), Hull (2005). A discrete American option is an American option with exercise dates being restricted to a discrete set of monitoring points; see Glasserman (2004). 
In this survey we shall focus on discrete barrier and lookback options, because very often they can be studied in similar ways, as their payoffs all depend on the extrema of the underlying stochastic processes. The study of discrete Asian options is of separate interest, and requires totally different techniques. Discrete American options are closely related to numerical pricing of American options; there is a separate survey in this handbook on them. Due to the similarity between discrete barrier options and discrete lookback options, we shall focus on discrete barrier options, although most of the techniques discussed here can be easily adapted to study discrete lookback options.

\subsection{Barrier and lookback options}

A standard (also called floating) lookback call (put) gives the option holder the right to buy (sell) an asset at its lowest (highest) price during the life of the option. In other words, the payoffs of the floating lookback call and put options are $S(T)-m_{0, T}$ and $M_{0, T}-S(T)$, respectively, where $m_{0, T}$ and $M_{0, T}$ are minimum and maximum of the asset price between 0 and $T$. In a discrete time setting the minimum (maximum) of the asset price will be determined at discrete monitoring instants. In the same way, the payoffs of the fixed strike put and call are $\left(K-m_{0, T}\right)^{+}$and $\left(M_{0, T}-K\right)^{+}$. Other types of lookback options include percentage lookback options in which the extreme values are multiplied by a constant, and partial lookback options in which the monitoring interval for the extremum is a subinterval of $[0, T]$. We shall refer the interested reader to Andreasen (1998) for a detailed description.

A barrier option is a financial derivative contract that is activated (knocked in) or extinguished (knocked out) when the price of the underlying asset (which could be a stock, an index, an exchange rate, an interest rate, etc.) crosses a certain level (called a barrier). For example, an up-and-out call option gives the option holder the payoff of a European call option if the price of the underlying asset does not reach a higher barrier level before the expiration date. More complicated barrier options may have two barriers (double barrier options), and may have the final payoff determined by one asset and the barrier level determined by another asset (two-dimensional barrier options); see Zhang (1998) and Hull (2005).

Taken together, discrete lookback and barrier options are among the most popular path-dependent options traded in exchanges worldwide and also in over-the-counter markets. Lookback and barrier options are also useful outside the context of literal options. For example, Longstaff (1995) approximates the values of marketability of a security over a fixed horizon with a type of continuous-time lookback option and gives a closed-form expression for the value; the discrete version of lookback options will be relevant in his setting. Merton (1974), Black and Cox (1976), and more recently Leland and Toft (1996), Rich (1996), and Chen and Kou (2005) among others, have used barrier models for study credit risk and pricing contingent claims with endogenous 
default. For tractability, this line of work typically assumes continuous monitoring of a reorganization boundary. But to the extent that the default can be modeled as a barrier crossing, it is arguably one that can be triggered only at the specific dates - e.g. coupon payment dates.

An important issue of pricing barrier options is whether the barrier crossing is monitored in continuous time or in discrete time. Most models assume the continuous time version mainly because this leads to analytical solutions; see, for example, Gatto et al. (1979), Goldman et al. (1979), and Conze and Viswanathan (1991), Heynen and Kat (1995) for continuous lookback options; and see, for example, Merton (1973), Heynen and Kat (1994a, 1994b), Rubinstein and Reiner (1991), Chance (1994), and Kunitomo and Ikeda (1992) for various formulae for continuously monitored barrier options under the classical Brownian motion framework. Recently, Boyle and Tian (1999) and Davydov and Linetsky (2001) have priced continuously monitored barrier and lookback options under the CEV model using lattice and Laplace transform methods, respectively; see Kou and Wang $(2003,2004)$ for continuously monitored barrier options under a jump-diffusion framework.

However in practice most, if not all, barrier options traded in markets are discretely monitored. In other words, they specify fixed times for monitoring of the barrier (typically daily closings). Besides practical implementation issues, there are some legal and financial reasons why discretely monitored barrier options are preferred to continuously monitored barrier options. For example, some discussions in trader's literature ("Derivatives Week", May 29th, 1995 ) voice concern that, when the monitoring is continuous, extraneous barrier breach may occur in less liquid markets while the major western markets are closed, and may lead to certain arbitrage opportunities.

Although discretely monitored barrier and lookback options are popular and important, pricing them is not as easy as that of their continuous counterparts for several reasons:

(1) There are essentially no closed solutions, except using $m$-dimensional normal distribution functions ( $m$ is the number of monitoring points), which cannot be computed easily if, for example, $m>5$; see Section 3 .

(2) Direct Monte Carlo simulation or standard binomial trees may be difficult, and can take hours to produce accurate results; see Broadie et al. (1999).

(3) Although the central limit theorem asserts that as $m \rightarrow \infty$ the difference between the discretely and continuously monitored barrier options should be small, it is well known that the numerical differences can be surprisingly large, even for large $m$; see, e.g., the table in Section 4.

Because of these difficulties, many numerical methods have been proposed for pricing discrete barrier and lookback options. 


\subsection{Overview of different methods}

First of all, by using the change of numeraire argument the pricing of barrier and lookback options can be reduced to studying either the marginal distribution of the first passage time, or the joint probability of the first passage time and the terminal value of a discrete random walk; see Section 2. Although there are many representations available for these two classical problems, there is little literature on how to compute the joint probability explicitly before the recent interest in discrete barrier and lookback options. Many numerical methods have been developed in the last decade for discrete barrier and lookback options. Popular ones are:

(1) Methods based on convolution, e.g. the fast Gaussian transform method developed in Broadie and Yamamoto (2003) and the Hilbert transform method in Feng and Linetsky (2005). This is basically due to the fact that the joint probability of the first passage time and the terminal value of a discrete random walk can be written as $m$-dimensional probability distribution (hence a $m$-dimensional integral or convolution.) We will review these results in Section 3.

(2) Methods based on the asymptotic expansion of discrete barrier options in terms of continuous barrier options, assuming $m \rightarrow \infty$. Of course, as we mentioned, the straightforward result from the central limit theorem, which has error $o(1)$, does not give a good approximation. An approximation based on the results from sequential analysis (see, e.g., Siegmund, 1985 with the error order $o(1 / \sqrt{m})$ is given in Broadie et al. (1999), whose proof is simplified in Kou (2003) and Hörfelt (2003). We will review these results in Section 4.

(3) Methods based on the perturbation analysis of differential equations, leading to a higher order expansion with the error order $o(1 / \mathrm{m})$. This is investigated in Howison and Steinberg (2005) and Howison (2005). We will review these results in Section 5.

(4) Methods based on transforms. Petrella and Kou (2004) use Laplace transforms to numerically invert the Spitzer's identity associated with the first passage times. We will review these transform-based methods in Section 6.

Besides these specialized methods, there are also many "general methods," such as lattice methods, Monte Carlo simulation, etc. We call them general methods because in principle these methods can be applied to broader contexts, e.g. American options and other path-dependent options, not just for discrete barrier and lookback options. Broadly speaking, general methods will be less efficient than the methods which take advantage of the special structures of discrete barrier and lookback options. However, general methods are attractive if one wants to develop a unified numerical framework to price different types of options, not just discrete barrier and lookback options. Because of their generality, many methods could potentially belong to this category, and it is very difficult to give a comprehensive review for them. Below is only a short list of some of general methods. 
(a) Lattice methods are among the most popular methods in option pricing. It is well known that the straightforward binomial tree is not efficient in pricing discrete and lookback barrier options, due to the inefficiencies in computing discrete extreme values of the sample paths involved in the payoffs. Broadie et al. (1999) proposed an enhanced trinomial tree method which explicitly uses the continuity correction in Broadie et al. (1997) and a shift node. A Dirichlet lattice method based on the conditional distribution via Brownian bridge is given in Kuan and Webber (2003). Duan et al. (2003) proposed a method based on Markov chain, in combination of lattice, simulation, and the quadrature method. Other lattice methods include adjusting the position of nodes (Ritchken, 1995; Cheuk and Vorst, 1997; Tian, 1999) and refining branching near the barrier (Figlewski and Gao, 1999; Ahn et al., 1999). See also Babbs (1992), Boyle and Lau (1994), Hull and White (1993), Kat (1995).

(b) Another popular general method is Monte Carlo simulation. Because the barrier options may involve events (e.g. barrier crossing) with very small probabilities, the straightforward simulation may have large variances. Variance reduction techniques, notably importance sampling and conditional sampling methods using Brownian bridge, can be used to achieve significant variance reduction. Instead of giving a long list of related papers, we refer the reader to an excellent book by Glasserman (2004).

(c) Since the price of a discrete barrier option can be formulated as a solution of partial differential equation, one can use various finite difference methods; see Boyle and Tian (1998) and Zvan et al. (2000).

(d) Because the prices of a discrete barrier price can be written in terms of $m$-dimensional integrals, one can also use numerical integration methods. See Ait-Sahalia and Lai (1997, 1998), Sullivan (2000), Tse et al. (2001), Andricopoulos et al. (2003), and Fusai and Recchioni (2003).

\subsection{Outline of the survey}

Due to the page limit, this survey focuses on methods that takes into account of special structures of the discrete barrier and lookback options, resulting in more efficient algorithms but with narrower scopes. In particular, we shall survey the following methods

(1) Broadie-Yamamoto method based on the fast Gaussian transform; see Section 3.

(2) Feng-Linetsky method based on Hilbert transform; see Section 3.

(3) A continuity correction approximation; see Section 4.

(4) Howison-Steinberg approximation based on the perturbation method; see Section 5.

(5) A Laplace inversion method based on Spitzer's identity; see Section 6.

This survey also contains a new (more direct) derivation of the constant related to the continuity correction; see Appendix B. 
Because this is a survey article we shall focus on giving intuition and comparing different methods, rather than giving detailed proofs which can be found in individual papers. For example, when we discuss the continuity correction we use a picture to illustrate the idea, rather than giving a proof. When we present the Howison-Steinberg approximation we spend considerable time on the basic background of the perturbation method (so that people with only probabilistic background can understand the intuition behind the idea), rather than giving the mathematical details, which involve both the Spitzer function for Wiener-Hopf equations and can be found in the original paper by Howison and Steinberg (2005).

\section{A representation of barrier options via the change of numeraire argument}

We assume the price of the underlying asset $S(t), t \geqslant 0$, satisfies $S(t)=$ $S(0) \exp \{\mu t+\sigma B(t)\}$, where under the risk-neutral probability $P^{*}$, the drift is $\mu=r-\sigma^{2} / 2, r$ is the risk-free interest rate and $B(t)$ is a standard Brownian motion under $P^{*}$. In the continuously monitored case, the standard finance theory implies that the price of a barrier option will be the expectation, taken with respect to the risk-neutral measure $P^{*}$, of the discounted (with the discount factor being $e^{-r T}$ with $T$ the expiration date of the option) payoff of the option. For example, the price of a continuous up-and-out call option is given by

$$
V(H)=\mathrm{E}^{*}\left(e^{-r T}(S(T)-K)^{+} I(\tau(H, S)>T)\right),
$$

where $K \geqslant 0$ is the strike price, $H>S(0)$ is the barrier and, for any process $Y(t)$, the notation $\tau(x, Y)$ means that $\tau(x, Y):=\inf \{t \geqslant 0: Y(t) \geqslant x\}$. The other seven types of the barrier options can be priced similarly. In the Brownian motion framework, all eight types of the barrier options can be priced in closed forms; see Merton (1973).

In the discretely monitoring case, under the risk neutral measure $P^{*}$, at the $n$th monitoring point, $n \Delta t$, with $\Delta t=T / m$, the asset price is given by

$$
\begin{aligned}
S_{n} & =S(0) \exp \left\{\mu n \Delta t+\sigma \sqrt{\Delta t} \sum_{i=1}^{n} Z_{i}\right\}=S(0) \exp \left(W_{n} \sigma \sqrt{\Delta t}\right), \\
n & =1,2, \ldots, m,
\end{aligned}
$$

where the random walk $W_{n}$ is defined by

$$
W_{n}:=\sum_{i=1}^{n}\left(Z_{i}+\frac{\mu}{\sigma} \sqrt{\Delta t}\right),
$$

the drift is given by $\mu=r-\sigma^{2} / 2$, and the $Z_{i}$ 's are independent standard normal random variables. By analogy, the price of the discrete up-and-out-call 
option is given by

$$
\begin{aligned}
V_{m}(H) & =\mathrm{E}^{*}\left(e^{-r T}\left(S_{m}-K\right)^{+} I\left(\tau^{\prime}(H, S)>m\right)\right) \\
& =\mathrm{E}^{*}\left\{e^{-r T}\left(S_{m}-K\right)^{+} I\left\{\tau^{\prime}(a /(\sigma \sqrt{T}), W)>m\right\}\right\},
\end{aligned}
$$

where $a:=\log (H / S(0))>0, \tau^{\prime}(H, S)=\inf \left\{n \geqslant 1: S_{n} \geqslant H\right\}, \tau^{\prime}(x, W)=$ $\inf \left\{n \geqslant 1: W_{n} \geqslant x \sqrt{m}\right\}$.

For any probability measure $P$, let $\hat{P}$ be defined by

$$
\frac{\mathrm{d} \hat{P}}{\mathrm{~d} P}=\exp \left\{\sum_{i=1}^{m} a_{i} Z_{i}-\frac{1}{2} \sum_{i=1}^{m} a_{i}^{2}\right\},
$$

where the $a_{i}, i=1, \ldots, n$, are arbitrary constants, and the $Z_{i}$ 's are standard normal random variables under the probability measure $P$. Then a discrete Girsanov theorem (Karatzas and Shreve, 1991, p. 190) implies that under the probability measure $\hat{P}$, for every $1 \leqslant i \leqslant m, \hat{Z}_{i}:=Z_{i}-a_{i}$ is a standard normal random variable.

By using the discrete Girsanov theorem, we can represent the price of a discrete barrier options as a difference of two probabilities under different measures. This is called the change of numeraire argument; for a survey, see Schroder (1999). It is applied to the case of discrete barrier options by Kou (2003) and Hörfelt (2003) independently. However, the methods in Kou (2003) and Hörfelt (2003) lead to slightly different barrier correction formulae. To illustrate the change of numeraire argument for the discrete barrier options. Let us consider the case of the discrete up-and-out call option, as the other seven options can be treated similarly; see e.g. Haug (1999).

First note that

$$
\begin{aligned}
\mathrm{E}^{*} & \left(e^{-r T}\left(S_{m}-K\right)^{+} I\left(\tau^{\prime}(H, S)>m\right)\right) \\
= & \mathrm{E}^{*}\left(e^{-r T}\left(S_{m}-K\right) I\left(S_{m} \geqslant K, \tau^{\prime}(H, S)>m\right)\right) \\
= & \mathrm{E}^{*}\left(e^{-r T} S_{m} I\left(S_{m} \geqslant K, \tau^{\prime}(H, S)>m\right)\right) \\
& -K e^{-r T} P^{*}\left(S_{m} \geqslant K, \tau^{\prime}(H, S)>m\right) .
\end{aligned}
$$

Using the discrete Girsanov theorem with $a_{i}=\sigma \sqrt{\Delta t}$, we have that the first term in the above equation is given by

$$
\begin{aligned}
\mathrm{E}^{*}\left[e^{-r T} S(0) \exp \left\{\mu m \Delta t+\sigma \sqrt{\Delta t} \sum_{i=1}^{m} Z_{i}\right\} I\left(S_{m} \geqslant K, \tau^{\prime}(H, S)>m\right)\right] \\
\quad=S(0) \mathrm{E}^{*}\left[\exp \left\{-\frac{1}{2} \sigma^{2} T+\sigma \sqrt{\Delta t} \sum_{i=1}^{m} Z_{i}\right\} I\left(S_{m} \geqslant K, \tau^{\prime}(H, S)>m\right)\right] \\
=S(0) \hat{\mathrm{E}}\left(I\left(S_{m} \geqslant K, \tau^{\prime}(H, S)>m\right)\right) \\
=S(0) \hat{P}\left(S_{m} \geqslant K, \tau^{\prime}(H, S)>m\right) .
\end{aligned}
$$


Under $\hat{P}, \log S_{m}$ has a mean $\mu m \Delta t+\sigma \sqrt{\Delta t} \cdot m \sigma \sqrt{\Delta t}=\left(\mu+\sigma^{2}\right) T$ instead of $\mu T$ under the measure $P^{*}$. Therefore, the price of a discrete up-and-out-call option is given by

$$
\begin{aligned}
V_{m}(H)= & S(0) \hat{P}\left(W_{m} \geqslant \frac{\log (K / S(0))}{\sigma \sqrt{\Delta t}}, \tau^{\prime}(a /(\sigma \sqrt{T}), W)>m\right) \\
& -K e^{-r T} P^{*}\left(W_{m} \geqslant \frac{\log (K / S(0))}{\sigma \sqrt{\Delta t}}, \tau^{\prime}(a /(\sigma \sqrt{T}), W)>m\right),
\end{aligned}
$$

where

$$
\text { under } \hat{P}, \quad \begin{aligned}
W_{m} & =\sum_{i=1}^{m}\left(\hat{Z}_{i}+\left\{\left(\mu+\sigma^{2}\right) / \sigma\right\} \sqrt{\frac{T}{m}}\right) \\
& =\sum_{i=1}^{m}\left(\hat{Z}_{i}+\left\{\left(r+\frac{1}{2} \sigma^{2}\right) / \sigma\right\} \sqrt{\frac{T}{m}}\right)
\end{aligned}
$$

and

$$
\text { under } \begin{aligned}
P^{*}, \quad W_{m} & =\sum_{i=1}^{m}\left(Z_{i}+(\mu / \sigma) \sqrt{\frac{T}{m}}\right) \\
& =\sum_{i=1}^{m}\left(Z_{i}+\left\{\left(r-\frac{1}{2} \sigma^{2}\right) / \sigma\right\} \sqrt{\frac{T}{m}}\right)
\end{aligned}
$$

with $\hat{Z}_{i}$ and $Z_{i}$ being standard normal random variables under $\hat{P}$ and $P^{*}$, respectively.

Therefore, the problem of pricing discrete barrier options is reduced to studying the joint probability of the first passage time $\left(\tau^{\prime}\right)$ and the terminal values $\left(W_{m}\right)$ of a discrete random walk. Note that we have a first passage problem for the random walk $W_{n}$ with a small drift $\left(\frac{\mu}{\sigma} \sqrt{\Delta t} \rightarrow 0\right.$, as $\left.m \rightarrow \infty\right)$ to cross a high boundary $(a \sqrt{m} /(\sigma \sqrt{T}) \rightarrow \infty$, as $m \rightarrow \infty)$.

\section{Convolution, Broadie-Yamamoto method via the fast Gaussian transform, and Feng-Linetsky method via Hilbert transform}

As we have seen in the last section, under the geometric Brownian motion model the prices of discrete barrier options can be represented as probabilities of random walk with increments having normal distributions. Thus, in principle analytical solutions of discrete barrier options can be derived using multivariate normal distributions; see, e.g., Heynan and Kat (1995) and Reiner (2000).

To give an illustration of the idea, consider a discrete up-and-in call option with two monitoring points, $t_{1}=T / 3, t_{2}=2 T / 3$, and $H<K$. Note that the 
maturity $T$ is not a monitoring point. We have

$$
\begin{aligned}
V_{3}(H)= & S(0) N_{2}\left(\hat{a}_{1, H,-}, \hat{a}_{K} ; \sqrt{t_{1} / T}\right) \\
& -K e^{-r T} N_{2}\left(a_{1, H,-}^{*}, a_{K}^{*} ; \sqrt{t_{1} / T}\right) \\
& +S(0) N_{3}\left(\hat{a}_{1, H,+}, \hat{a}_{2, H,-}, \hat{a}_{K} ;-\sqrt{t_{1} / t_{2}},-\sqrt{t_{1} / T}, \sqrt{t_{2} / T}\right) \\
& -K e^{-r T} N_{3}\left(a_{1, H,+}^{*}, a_{2, H,-}^{*}, a_{K}^{*} ;-\sqrt{t_{1} / t_{2}},-\sqrt{t_{1} / T}, \sqrt{t_{2} / T}\right),
\end{aligned}
$$

where the constants are

$$
\begin{aligned}
& \hat{a}_{1, H, \pm} \equiv \frac{ \pm \log (H / S(0))-\left\{\left(r+\frac{1}{2} \sigma^{2}\right)\right\} t_{1}}{\sigma \sqrt{t_{1}}}, \\
& a_{1, H, \pm}^{*} \equiv \frac{ \pm \log (H / S(0))-\left\{\left(r-\frac{1}{2} \sigma^{2}\right)\right\} t_{1}}{\sigma \sqrt{t_{1}}} \\
& \hat{a}_{2, H, \pm} \equiv \frac{ \pm \log (H / S(0))-\left\{\left(r+\frac{1}{2} \sigma^{2}\right)\right\} t_{2}}{\sigma \sqrt{t_{2}}}, \\
& a_{2, H, \pm}^{*} \equiv \frac{ \pm \log (H / S(0))-\left\{\left(r-\frac{1}{2} \sigma^{2}\right)\right\} t_{2}}{\sigma \sqrt{t_{2}}}, \\
& \hat{a}_{K} \equiv \frac{-\log (K / S(0))-\left\{\left(r+\frac{1}{2} \sigma^{2}\right)\right\} T}{\sigma \sqrt{T}}, \\
& a_{K}^{*} \equiv \frac{-\log (K / S(0))-\left\{\left(r-\frac{1}{2} \sigma^{2}\right)\right\} T}{\sigma \sqrt{T}} .
\end{aligned}
$$

The proof of (1) is given ir Appendix A. Here $N_{2}$ and $N_{3}$ denote the standard bivariate and trivariate normal distributions:

$$
N_{2}\left(z_{1}, z_{2} ; \varrho\right)=P\left(Z_{1} \leqslant z_{1}, Z_{2} \leqslant z_{2}\right),
$$

where $Z_{1}$ and $Z_{2}$ are standard bivariate normal random variables with correlation $\varrho$, and

$$
N_{3}\left(z_{1}, z_{2}, z_{3} ; \varrho_{12}, \varrho_{13}, \varrho_{23}\right)=P\left(Z_{1} \leqslant z_{1}, Z_{2} \leqslant z_{2}, Z_{3} \leqslant z_{3}\right),
$$

with correlations $\varrho_{12}, \varrho_{13}, \varrho_{23}$. The pricing formula in (1) can be easily generalized to the case of $m$ (not necessarily equally spaced) monitoring points, so that the price of a discrete barrier option with $m$ monitoring points can be written involving the sum of multivariate normal distribution functions, with the highest dimension in the multivariate normal distributions being $m$.

However, $m$-dimensional normal distribution functions can hardly be computed easily if, for example, $m>5$. Reiner (2000) proposed to use the fast Fourier transform to compute the convolution in the multivariate normal distribution. Recently there are two powerful ways to evaluate the convolution. One is the fast Gaussian transform in Broadie and Yamamoto (2003) in which 
the convolution is computed very fast under the Gaussian assumption. The second method is the Hilbert transform method in Feng and Linetsky (2005), in which they recognize an interesting linking between Fourier transform of indicator functions and Hilbert transform. Feng and Linetsky method is more general, as it works as long as the asset returns follow a Lévy process. Below we give a brief summary of the two methods.

\subsection{Broadie-Yamamoto method via the fast Gaussian transform}

One of the key idea of Broadie-Yamamoto method is to recognize that one can compute integrals in convolution very fast if the integrals only involves normal density. For example, consider the discrete sum of Gaussian densities.

$$
A\left(x_{m}\right)=\sum_{n=1}^{N} w_{n} \exp \left\{-\frac{\left(x_{m}-y_{n}\right)^{2}}{\delta}\right\}, \quad i=1, \ldots, M .
$$

The direct computation of the above sums will need $O(N M)$ operations. However, by using the Hermite functions to approximate the Gaussian densities, one can perform the above sum in $O(N)+O(1)+O(M)=O(\max (N, M))$ operations.

More precisely, the Hermite expansion yields

$$
\begin{aligned}
\exp \left\{-\frac{\left(x_{m}-y_{n}\right)^{2}}{\delta}\right\}= & \sum_{i=1}^{\infty} \sum_{j=1}^{\infty} \frac{1}{i ! j !}\left(\frac{y_{n}-y_{0}}{\sqrt{\delta}}\right)^{j}\left(\frac{x_{m}-x_{0}}{\sqrt{\delta}}\right)^{i} \\
& \times H_{i+j}\left(\frac{x_{0}-y_{0}}{\sqrt{\delta}}\right),
\end{aligned}
$$

where $H_{i+j}(\cdot)$ is the Hermite function. The expansion converges quite fast, typically eight terms may be enough. In other words, we have an approximation

$$
\begin{aligned}
\exp \left\{-\frac{\left(x_{m}-y_{n}\right)^{2}}{\delta}\right\} \approx & \sum_{i=1}^{\alpha_{\max }} \sum_{j=1}^{\alpha_{\max }} \frac{1}{i ! j !}\left(\frac{y_{n}-y_{0}}{\sqrt{\delta}}\right)^{j}\left(\frac{x_{m}-x_{0}}{\sqrt{\delta}}\right)^{i} \\
& \times H_{i+j}\left(\frac{x_{0}-y_{0}}{\sqrt{\delta}}\right),
\end{aligned}
$$

where $\alpha_{\max }$ is a small number, say no more than 8 . Using this approximation, we have the Gaussian sum is given by

$$
\begin{aligned}
A\left(x_{m}\right) & \approx \sum_{n=1}^{N} w_{n} \sum_{i=1}^{\alpha_{\max }} \sum_{j=1}^{\alpha_{\max }} \frac{1}{i ! j !}\left(\frac{y_{n}-y_{0}}{\sqrt{\delta}}\right)^{j}\left(\frac{x_{m}-x_{0}}{\sqrt{\delta}}\right)^{i} H_{i+j}\left(\frac{x_{0}-y_{0}}{\sqrt{\delta}}\right) \\
& =\sum_{i=1}^{\alpha_{\max }} \frac{1}{i !}\left[\sum_{j=1}^{\alpha_{\max }} \frac{1}{j !}\left\{\sum_{n=1}^{N} w_{n}\left(\frac{y_{n}-y_{0}}{\sqrt{\delta}}\right)^{j}\right\} H_{i+j}\left(\frac{x_{0}-y_{0}}{\sqrt{\delta}}\right)\right]
\end{aligned}
$$




$$
\times\left(\frac{x_{m}-x_{0}}{\sqrt{\delta}}\right)^{i} \text {. }
$$

Now the algorithm becomes

1. Compute $B_{j}=\sum_{n=1}^{N} w_{n}\left(\frac{y_{n}-y_{0}}{\sqrt{\delta}}\right)^{j}$ for $j=1, \ldots, \alpha_{\max }$.

2. Compute $C_{i}=\sum_{j=1}^{\alpha_{\max }} \frac{1}{j !} B_{j} H_{i+j}\left(\frac{x_{0}-y_{0}}{\sqrt{\delta}}\right)$ for $i=1, \ldots, \alpha_{\max }$.

3. Approximate $A\left(x_{m}\right)$ as $\sum_{i=1}^{\alpha_{\max }} \frac{1}{i !} C_{i}\left(\frac{x_{m}-x_{0}}{\sqrt{\delta}}\right)^{i}$ for $m=1, \ldots, M$.

When $\alpha_{\max }$ is fixed, the total number of operations is therefore $O(N)+$ $O(1)+O(M)=O(\max (N, M))$. Broadie and Yamamoto (2003) show that the above fast Gaussian transform is very fast. In fact, it is perhaps the fastest algorithm we can get so far under the Gaussian assumption. Of course, the algorithm relies on the special structure of the Gaussian distribution. For other distributions, similar algorithms might be available if some fast and accurate expansions of the density functions are available.

\subsection{Feng-Linetsky method via Hilbert transform}

Feng and Linetsky (2005) proposed a novel method to compute the convolution related to discrete barrier options via Hilbert transform. The key idea is that multiplying a function with the indicator function in the state space corresponds to Hilbert transform in the Fourier space. The method computes a sequence of Hilbert transforms at the discrete monitoring points, and then conducts one final Fourier inversion to get the option price. Feng-Linetsky method is quite general, as it works in principle for any Lévy process and for both single and double barrier options. The method also works very fast, as the number of operations is $O\left(M N \log _{2} N\right)$, where $M$ is the number of monitoring points and $N$ is the number of sample points needed to compute the Hilbert transform.

To get an intuition of the idea, we shall illustrate a basic version of the method in terms of computing the probability $p(x)$ for a standard Brownian motion $B(t)$

$$
\begin{aligned}
p(x) & =P\left(\min \left\{B_{\Delta}, B_{2 \Delta}, \ldots, B_{M \Delta}\right\}>0 \mid B_{0}=x\right) \\
& =\mathrm{E}\left\{\prod_{i=1}^{M} I\left[B_{i \Delta}>0\right] \mid B_{0}=x\right\} .
\end{aligned}
$$

We can compute $p(x)$ by the backward induction

$$
\begin{aligned}
& v^{M}(x)=I(x>0), \\
& \begin{aligned}
v^{M-1}(x) & =I(x>0) \cdot \mathrm{E}\left\{I\left(B_{\Delta}>0\right) \mid B_{0}=x\right\} \\
& =I(x>0) \cdot \mathrm{E}\left\{v^{M}\left(B_{\Delta}\right) \mid B_{0}=x\right\},
\end{aligned}
\end{aligned}
$$




$$
\begin{aligned}
& v^{M-2}(x)=I(x>0) \cdot \mathrm{E}\left\{I\left(B_{\Delta}>0\right) I\left(B_{2 \Delta}>0\right) \mid B_{0}=x\right\} \\
& =I(x>0) \cdot \mathrm{E}\left\{v^{M-1}\left(B_{\Delta}\right) \mid B_{0}=x\right\}, \\
& p(x)=\mathrm{E}\left\{v^{1}\left(B_{\Delta}\right) \mid B_{0}=x\right\} .
\end{aligned}
$$

To take a Fourier transform we introduce a rescaling factor $e^{\alpha x}$,

$$
v_{\alpha}^{j}(x)=e^{\alpha x} v^{j}(x), \quad \alpha<0,
$$

because the indicator function $I(x>0)$ is not a $L^{1}$ function. This is equivalent to perform a Laplace transform. The backward induction becomes

$$
\begin{aligned}
v_{\alpha}^{M}(x)= & e^{\alpha x} I(x>0), \\
v_{\alpha}^{M-1}(x) & =e^{\alpha x} \cdot I(x>0) \cdot \mathrm{E}\left\{I\left(B_{\Delta}>0\right) \mid B_{0}=x\right\} \\
& =I(x>0) \cdot \mathrm{E}\left\{e^{-\alpha\left(B_{\Delta}-x\right)} e^{\alpha B_{\Delta}} I\left(B_{\Delta}>0\right) \mid B_{0}=x\right\} \\
& =I(x>0) \cdot \mathrm{E}\left\{e^{-\alpha\left(B_{\Delta}-x\right)} v_{\alpha}^{M}\left(B_{\Delta}\right) \mid B_{0}=x\right\} \\
& =e^{\Delta \alpha^{2} / 2} \cdot I(x>0) \cdot \mathrm{E}\left\{e^{-\Delta \alpha^{2} / 2} e^{-\alpha\left(B_{\Delta}-x\right)} v_{\alpha}^{M}\left(B_{\Delta}\right) \mid B_{0}=x\right\} \\
& =e^{\Delta \alpha^{2} / 2} \cdot I(x>0) \cdot \mathrm{E}_{-\alpha}\left\{v_{\alpha}^{M}\left(B_{\Delta}\right) \mid B_{0}=x\right\},
\end{aligned}
$$

where $E_{-\alpha}$ means Brownian motion with drift $-\alpha$ and the last equality follows from Girsanov theorem. Similarly,

$$
\begin{aligned}
v_{\alpha}^{M-2}(x)= & e^{\alpha x} \cdot I(x>0) \cdot \mathrm{E}\left\{I\left(B_{\Delta}>0\right) I\left(B_{2 \Delta}>0\right) \mid B_{0}=x\right\} \\
= & I(x>0) \cdot \mathrm{E}\left\{e^{-\alpha\left(B_{\Delta}-x\right)} I\left(B_{\Delta}>0\right)\right. \\
& \left.\cdot \mathrm{E}\left\{e^{-\alpha\left(B_{2 \Delta}-B_{\Delta}\right)} v^{M}\left(B_{2 \Delta}\right) \mid B_{\Delta}\right\} \mid B_{0}=x\right\} \\
= & I(x>0) \cdot \mathrm{E}\left\{e^{-\alpha\left(B_{\Delta}-x\right)} v^{M-1}\left(B_{\Delta}\right) \mid B_{0}=x\right\} \\
= & e^{\Delta \alpha^{2} / 2} I(x>0) \cdot \mathrm{E}\left\{e^{-\Delta \alpha^{2} / 2} e^{-\alpha\left(B_{\Delta}-x\right)} v^{M-1}\left(B_{\Delta}\right) \mid B_{0}=x\right\} \\
= & e^{\Delta \alpha^{2} / 2} I(x>0) \cdot \mathrm{E}_{-\alpha}\left\{v_{\alpha}^{M-1}\left(B_{\Delta}\right) \mid B_{0}=x\right\} .
\end{aligned}
$$

In general, we have a backward induction

$$
\begin{aligned}
& v_{\alpha}^{M}(x)=e^{\alpha x} I(x>0), \\
& v_{\alpha}^{j-1}(x)=e^{\Delta \alpha^{2} / 2} I(x>0) \cdot \mathrm{E}_{-\alpha}\left\{v^{j}\left(B_{\Delta}\right) \mid B_{0}=x\right\}, \quad j=M, \ldots, 2, \\
& p(x)=e^{-\alpha x} e^{\Delta \alpha^{2} / 2} \cdot \mathrm{E}_{-\alpha}\left\{v^{1}\left(B_{\Delta}\right) \mid B_{0}=x\right\} .
\end{aligned}
$$

Denote $\hat{v}_{\alpha}^{j}(x)$ to be the Fourier transform of $v_{\alpha}^{j}(x)$, which is possible as $e^{\alpha x} I(x>0)$ is a $L^{1}$ function. Now the Fourier transform in the backward induction will involve Fourier transform of product of the indicator function and another function. The key observation in Feng and Linetsky (2005) is that Fourier transform of the product of the indicator function and a function can 
be written in terms of Hilbert transform. More precisely,

$$
\mathcal{F}\left(I_{(0, \infty)} \cdot f\right)(\xi)=\frac{1}{2}(\mathcal{F} f)(\xi)+\frac{i}{2}(\mathcal{H} f)(\xi),
$$

where $\mathcal{F}$ denotes Fourier transform and $\mathcal{H}$ denotes Hilbert transform defined by the Cauchy principle value integral, i.e.

$$
(\mathcal{H} f)(\xi)=\frac{1}{\pi} P . V . \int_{-\infty}^{\infty} \frac{f(\eta)}{\xi-\eta} \mathrm{d} \eta .
$$

To compute $p(x)$ one needs to compute $M-1$ Hilbert transforms and then conducts one final Fourier inversion. As shown in Feng and Linetsky (2005), a Hilbert transform can be computed efficiently by using approximation theory in Hardy spaces which leads to a simple trapezoidal-like quadrature sum.

In general Feng-Linetsky method is slower than Broadie-Yamamoto method, if the underlying model is Gaussian (e.g. under Black-Scholes model or Merton (1976) normal jump diffusion model). For example, as it is pointed out in Feng and Linetsky (2005) it may take 0.01 seconds for Broadie-Yamamoto to achieve accuracy of $10^{-12}$ under the Black-Scholes model, while it may take 0.04 seconds for Feng-Linetsky method to achieve accuracy of $10^{-8}$. The beauty of Feng-Linetsky method is that it works for general Lévy processes with very reasonable computational time.

\section{Continuity corrections}

\subsection{The approximation}

Broadie et al. (1997) proposed a continuity correction for the discretely monitored barrier option, and justified the correction both theoretically and numerically (Chuang, 1996 independently suggested the approximation in a heuristic way). The resulting approximation, which only relies on a simple correction to the Merton (1973) formula (thus trivial to implement), is nevertheless quite accurate and has been used in practice; see, for example, the textbook by Hull (2005).

More precisely, let $V(H)$ be the price of a continuous barrier option, and $V_{m}(H)$ be the price of an otherwise identical barrier option with $m$ monitoring points. Then for any of the eight discrete monitored regular barrier options the approximation is

$$
V_{m}(H)=V\left(H e^{ \pm \beta \sigma \sqrt{T / m}}\right)+o(1 / \sqrt{m}),
$$

with + for an up option and - for a down option, where the constant $\beta=$ $-\frac{\zeta(1 / 2)}{\sqrt{2 \pi}} \approx 0.5826, \zeta$ the Riemann zeta function. The approximation (2) was 
Table 1.

Up-and-Out-Call Option Price Results, $m=50$ (daily monitoring).

\begin{tabular}{lllll}
\hline Barrier & $\begin{array}{l}\text { Continuous } \\
\text { barrier }\end{array}$ & $\begin{array}{l}\text { Corrected } \\
\text { barrier, } \\
\text { Eq. (2) }\end{array}$ & True & $\begin{array}{l}\text { Relative error } \\
\text { of Eq. (2) } \\
\text { (in percent) }\end{array}$ \\
\hline 155 & 12.775 & 12.905 & 12.894 & 0.1 \\
150 & 12.240 & 12.448 & 12.431 & 0.1 \\
145 & 11.395 & 11.707 & 11.684 & 0.2 \\
140 & 10.144 & 10.581 & 10.551 & 0.3 \\
135 & 8.433 & 8.994 & 8.959 & 0.4 \\
130 & 6.314 & 6.959 & 6.922 & 0.5 \\
125 & 4.012 & 4.649 & 4.616 & 0.7 \\
120 & 1.938 & 2.442 & 2.418 & 1.0 \\
115 & 0.545 & 0.819 & 0.807 & 1.5 \\
\hline
\end{tabular}

This table is taken from Broadie et al. (1997, Table 2.6). The option parameters are $S(0)=110, K=$ $100, \sigma=0.30$ per year, $r=0.1$, and $T=0.2$ year, which represents roughly 50 trading days.

proposed in Broadie et al. (1997), where it is proved for four cases: downand-in call, down-and-out call, up-and-in put, and up-and-out put. Kou (2003) covered all eight cases with a simpler proof (see also Hörfelt, 2003). The continuity corrections for discrete lookback options are given in Broadie et al. (1999).

To get a feel of the accuracy of the approximation, Table 1 is taken from Broadie et al. (1997). The numerical results suggest that, even for daily monitored discrete barrier options, there can still be big differences between the discrete prices and the continuous prices. The improvement from using the approximation, which shifts the barrier from $H$ to $H e^{ \pm \beta \sigma \sqrt{T / m}}$ in the continuous time formulae, is significant.

Cao and Kou (2007) derived some barrier correction formulae for twodimensional barrier options and partial barrier options, which have some complications. For example, for a partial barrier option one cannot simply shifts the barrier up or down uniformly by a fixed constant, and one has to study carefully the different roles that the barrier plays in a partial barrier option; more precisely, the same barrier can sometimes be a terminal value, sometimes as a upcrossing barrier, and sometimes as a downcrossing barrier, all depending on what happens along the sample paths.

\subsection{Continuity correction for random walk}

The idea of continuity correction goes back to a classical technique in "sequential analysis," in which corrections to normal approximation are made to adjust for the "overshoot" effects when a discrete random walk crosses a barrier; see, for example, Chernoff (1965), Siegmund (1985), and Woodroofe (1982). 
For a standard Brownian motion $B(t)$ under any probability space $P$, define the stopping times for discrete random walk and for continuous-time Brownian motion as

$$
\begin{aligned}
& \tau^{\prime}(b, U):=\inf \left\{n \geqslant 1: U_{n} \geqslant b \sqrt{m}\right\}, \\
& \tilde{\tau}^{\prime}(b, U):=\inf \left\{n \geqslant 1: U_{n} \leqslant b \sqrt{m}\right\}, \\
& \tau(b, U):=\inf \{t \geqslant 0: U(T) \geqslant b\}, \\
& \tilde{\tau}(b, U):=\inf \{t \geqslant 0: U(T) \leqslant b\} .
\end{aligned}
$$

Here $U(t):=v t+B(t)$ and $U_{n}$ is a random walk with a small drift (as $m \rightarrow \infty$ ), $U_{n}:=\sum_{i=1}^{n}\left(Z_{i}+\frac{v}{\sqrt{m}}\right)$, where the $Z_{i}$ 's are independent standard normal random variables under $P$. Note that for general Lévy processes, we have the discrete random increments $Z_{i}$ 's are independent standard random variables, not necessarily normally distributed under $P$. In the case of Brownian motion, the approximation comes from a celebrated result in sequential analysis (Siegmund and Yuh, 1982; Siegmund, 1985, pp. 220-224): For any constants $b \geqslant y$ and $b>0$, as $m \rightarrow \infty$,

$$
\begin{aligned}
& P\left(U_{m}<y \sqrt{m}, \tau^{\prime}(b, U) \leqslant m\right) \\
& \quad=P(U(1) \leqslant y, \tau(b+\beta / \sqrt{m}, U) \leqslant 1)+o(1 / \sqrt{m}) .
\end{aligned}
$$

Here the constant $\beta$ is the limiting expectation of the overshoot,

$$
\beta=\frac{\mathrm{E}\left(A_{N}^{2}\right)}{2 \mathrm{E}\left(A_{N}\right)},
$$

where the mean zero random walk $A_{n}$ is defined as $A_{n}:=\sum_{i=1}^{n} Z_{i}$, and $N$ is the first ladder height associated with $A_{n}, N=\min \left\{n \geqslant 1: A_{n}>0\right\}$. For general Lévy processes, there will be some extra terms in addition to the constant $\beta$.

\subsubsection{An intuition via the reflection principle}

To get an intuition of (3), we consider the reflection principle for the standard Brownian motions when the drift $v=0$. The general case with a nonzero drift can be handled by using the likelihood ratio method. The reflection principle (see, e.g., Karatzas and Shreve, 1991) for the standard Brownian motion yields that

$$
P(U(1) \leqslant y, \tau(b, U) \leqslant 1)=P(U(1) \geqslant 2 b-y) .
$$

Intuitively, due to the random overshoot $R_{m}:=U_{\tau^{\prime}}-b \sqrt{m}$, the reflection principle for random walk should be

$$
P\left(U_{m}<y \sqrt{m}, \tau^{\prime}(b, U) \leqslant m\right)=P\left(U_{m} \geqslant 2\left(b \sqrt{m}+R_{m}\right)-y \sqrt{m}\right) .
$$

See Fig. 1 for an illustration. 




Fig. 1. An Illustration of the Heuristic Reflection Principle.

Replacing the random variable $R_{m}$ by its is expectation $\mathrm{E}\left(R_{m}\right)$ and using the fact from the renewal theory that

$$
\mathrm{E}\left(R_{m}\right) \rightarrow \frac{\mathrm{E}\left(A_{N}^{2}\right)}{2 \mathrm{E}\left(A_{N}\right)}=\beta,
$$

we have

$$
\begin{aligned}
& P\left(U_{m}<y \sqrt{m}, \tau^{\prime}(b, U) \leqslant m\right) \\
& \quad \approx P\left(U_{m} \geqslant\left\{2\left(b+\frac{\beta}{\sqrt{m}}\right)\right\} \sqrt{m}-y \sqrt{m}\right) \\
& \quad \approx P\left(U(1) \geqslant 2\left(b+\frac{\beta}{\sqrt{m}}\right)-y\right) \\
& =P(U(1) \leqslant y, \tau(b+\beta / \sqrt{m}, U) \leqslant 1),
\end{aligned}
$$

thus providing an intuition for (3).

\subsubsection{Calculating the constant $\beta$}

For any independent identically distributed random variables $Z_{i}$ with mean zero and variance one there are two ways to compute $\beta$, one by infinite series and the other a one-dimensional integral.

In the first approach, we have the following result from Spitzer (1960) about $\mathrm{E}\left(A_{N}\right)$ :

$$
\mathrm{E}\left(A_{N}\right)=\frac{1}{\sqrt{2}} e^{\omega_{0}},
$$


and from Lai (1976) about $\mathrm{E}\left(A_{N}^{2}\right)$ :

$$
\mathrm{E}\left(A_{N}^{2}\right)=\left\{\omega_{2}+\frac{\mathrm{E}\left(Z_{1}^{3}\right)}{3 \sqrt{2}}-\sqrt{2} \omega_{1}\right\} e^{\omega_{0}},
$$

where

$$
\begin{aligned}
& \omega_{0}=\sum_{n=1}^{\infty} \frac{1}{n}\left(P\left\{A_{n} \leqslant 0\right\}-\frac{1}{2}\right), \\
& \omega_{2}=1-\frac{1}{\sqrt{\pi}} \sum_{n=1}^{\infty}\left\{\frac{1}{\sqrt{n}}-\sqrt{\pi}\left(\begin{array}{c}
-\frac{1}{2} \\
n
\end{array}\right)(-1)^{n}\right\}, \\
& \left(\begin{array}{l}
x \\
n
\end{array}\right)=x(x-1) \cdots(x-n+1) / n !, \\
& \omega_{1}=\sum_{n=1}^{\infty} \frac{1}{\sqrt{n}}\left(\mathrm{E}\left[\left(A_{n} / \sqrt{n}\right)^{-}\right]-\frac{1}{\sqrt{2 \pi}}\right) .
\end{aligned}
$$

In the special of normal random variables, an explicit calculation of $\beta$ is available. Indeed in this case we have $\omega_{0}=0, \omega_{1}=0, E\left(Z_{1}^{3}\right)=0$, whence

$$
\beta=\frac{\mathrm{E}\left(A_{N}^{2}\right)}{2 \mathrm{E}\left(A_{N}\right)}=\frac{\left\{\omega_{2}+\frac{\mathrm{E}\left(Z_{1}^{3}\right)}{3 \sqrt{2}}-\sqrt{2} \omega_{1}\right\} e^{\omega_{0}}}{2 \frac{1}{\sqrt{2}} e^{\omega_{0}}}=\frac{\omega_{2}}{\sqrt{2}} .
$$

In Appendix B, we shall prove in the case of normal random variables, i.e. the Brownian model,

$$
\beta=\frac{\mathrm{E}\left(A_{N}^{2}\right)}{2 \mathrm{E}\left(A_{N}\right)}=\frac{\omega_{2}}{\sqrt{2}}=-\frac{\zeta(1 / 2)}{\sqrt{2 \pi}}
$$

with $\zeta$ being Riemann zeta function. Comparing to the existing proofs, the proof in Appendix B appears to be more direct and is new.

The link between $\beta$ and the Riemann zeta function as in (5) has been noted by Chernoff (1965) in an optimal stopping problem via Wiener-Hopf integral equations. The links between Wiener-Hopf integral equations and the Riemann zeta function are advanced further by Howison and Steinberg (2005), who provide a very elegant second order expansion via the perturbation method and the Spitzer function. The proof that the constant calculated in Chernoff (1965) from Wiener-Hopf equations and the constant in Siegmund (1979) for the continuity correction are the same is given in Hogan (1986). Later Chang and Peres (1997) who give a much more general result regarding connections between ladder heights and Riemann zeta function in the case of normal random variables, which covers (5) as a special case. See also a related expansion in Blanchet and Glynn (2006), Asmussen et al. (1995). In Appendix B we shall prove (5) in the case of normal random variables directly without 
using the general results in Chang and Peres (1997) or appealing to the argument in Hogan (1986).

There is also another integral representation (Siegmund, 1985, p. 225) for $\beta$, if $Z_{1}$ is a continuous random variable:

$$
\beta=\frac{\mathrm{E}\left(Z_{1}^{3}\right)}{6}-\frac{1}{\pi} \int_{0}^{\infty} \frac{1}{\lambda^{2}} \operatorname{Re}\left\{\log \left(\frac{2\left(1-E\left(\exp \left\{i \lambda Z_{1}\right\}\right)\right)}{\lambda^{2}}\right)\right\} \mathrm{d} \lambda .
$$

In the case of normal random variables we have $\mathrm{E}\left(\exp \left\{i \lambda Z_{1}\right\}\right)=e^{-\lambda^{2} / 2}$, and

$$
\begin{aligned}
\beta & =-\frac{1}{\pi} \int_{0}^{\infty} \frac{1}{\lambda^{2}} \log \left(\frac{1-e^{-\lambda^{2} / 2}}{\lambda^{2} / 2}\right) \mathrm{d} \lambda \\
& =-\frac{1}{\pi \sqrt{2}} \int_{0}^{\infty} \frac{1}{x^{2}} \log \left(\frac{1-e^{-x^{2}}}{x^{2}}\right) \mathrm{d} x .
\end{aligned}
$$

It is shown by Comtet and Majumdar (2005) that

$$
\frac{1}{\pi} \int_{0}^{\infty} \frac{1}{x^{2}} \log \left(\frac{1-e^{-x^{\alpha}}}{x^{\alpha}}\right) \mathrm{d} x=\frac{\zeta(1 / \alpha)}{(2 \pi)^{1 / \alpha} \sin \left(\frac{\pi}{2 \alpha}\right)}, \quad 1<\alpha \leqslant 2 .
$$

In particular, letting $\alpha=2$ yields

$$
\frac{1}{\pi} \int_{0}^{\infty} \frac{1}{x^{2}} \log \left(\frac{1-e^{-x^{2}}}{x^{2}}\right) \mathrm{d} x=-\frac{\zeta(1 / 2)}{(2 \pi)^{1 / 2} \sin \left(\frac{\pi}{4}\right)}=\frac{\zeta(1 / 2)}{(\pi)^{1 / 2}},
$$

and

$$
\beta=-\frac{1}{\pi \sqrt{2}} \int_{0}^{\infty} \frac{1}{x^{2}} \log \left(\frac{1-e^{-x^{2}}}{x^{2}}\right) \mathrm{d} x=-\frac{\zeta(1 / 2)}{\sqrt{2 \pi}} .
$$

Comtet and Majumdar (2005) also evaluated (6) for other symmetric distributions, such as symmetric Laplace and uniform distributions.

\subsubsection{A difficulty in generalization}

The above theory of continuity correction depends crucially on the idea of asymptotic analysis of a random walk (in our case $\sum_{i=1}^{n} Z_{i}$ ) indexed by a single exponential family of random variables. In our case the exponential family has a base of $N(0,1)$ related to $Z_{i}$ and the members in the family being $Z_{i}+v / \sqrt{m}$ with a distribution $N(v / \sqrt{m}, 1)$. In the general case, such as jump diffusion models, it is not clear how to write down a formula for the continuity correction for an exponential family of distribution indexed by a single parameter, because there could be several sources of randomness (Brownian parts, jump parts, etc.) and several parameters involved. 


\section{Perturbation method}

Since the price of a discrete barrier option can be written as a solution to a partial differential equation (PDE) with piecewise linear boundary conditions, numerical techniques from PDEs are also useful. One particular numerical technique is the perturbation method, which formally matches various asymptotic expansions to get approximations. This has been used by Howison and Steinberg (2005), Howison (2005) to get very accurate approximation for discrete barrier and American options.

\subsection{Basic concept of the perturbation method}

The perturbation method first identifies a parameter to be small so that approximations can be made around the zero value of the parameter. In fact the perturbation method will identify two solutions, inner and outer solutions, to match boundary conditions. The final approximation is a sum of both solutions minus a matching constant. To illustrate the basic idea of the perturbation method, let us consider an ordinary differential equation

$$
\varepsilon y^{\prime \prime}+y^{\prime}=t, \quad 0<t<1 ; \quad y(0)=y(1)=1,
$$

where the parameter $\varepsilon$ is a small number. If we let $\varepsilon=0$, then we get a solution $y=t^{2} / 2+C$. However this solution cannot satisfy both boundary conditions $y(0)=y(1)=1$. To get around with this difficulty, we shall have two solutions, one near 0 (inner solution) and one near 1 (outer solution) so that the final approximation can properly combine the two (called "matching").

The outer solution is given by setting $\varepsilon=0$ and matching the value at the right boundary,

$$
y_{1}(t)=\frac{t^{2}}{2}+\frac{1}{2}, \quad y_{1}(1)=1 .
$$

For the inner solution we can rescale the time, as we are more interested in what happen around $t=0$. Using $s=t / \varepsilon$ and $A(s)=y(t)$, we have a rescaled equation

$$
\frac{\varepsilon}{\varepsilon^{2}} \frac{\mathrm{d}^{2} A}{\mathrm{~d} s^{2}}+\frac{1}{\varepsilon} \frac{\mathrm{d} A}{\mathrm{~d} s}=\varepsilon s, \quad \text { or } \quad \frac{\mathrm{d}^{2} A}{\mathrm{~d} s^{2}}+\frac{\mathrm{d} A}{\mathrm{~d} s}=\varepsilon^{2} s .
$$

Letting $\varepsilon=0$ yields a linear ordinary differential equation,

$$
\frac{\mathrm{d}^{2} A}{\mathrm{~d} s^{2}}+\frac{\mathrm{d} A}{\mathrm{~d} s}=0
$$

which has a solution $A(s)=a+b e^{-s}$. Changing it back to $t$ we have the inner solution $y_{2}(t)=a+b e^{-t / \varepsilon}$. Matching the boundary at 0 , we have

$$
y_{2}(t)=(1-b)+b e^{-t / \varepsilon}, \quad y_{2}(0)=1 \text {. }
$$


Next we need to choose $b$ to match the inner and outer solution at some immediate region after $t=0$. To do this we try $u=t / \sqrt{\varepsilon}$. Then

$$
\begin{aligned}
& y_{1}(u \sqrt{\varepsilon})=\frac{u^{2} \varepsilon}{2}+\frac{1}{2} \rightarrow \frac{1}{2}, \\
& y_{2}(u \sqrt{\varepsilon})=(1-b)+b e^{-u \sqrt{\varepsilon} / \varepsilon} \rightarrow 1-b,
\end{aligned}
$$

yielding that $1-b=1 / 2$ or $b=1 / 2$. In summary the outer and inner solutions are

$$
y_{1}(t)=\frac{t^{2}}{2}+\frac{1}{2}, \quad y_{2}(t)=\frac{1}{2}+\frac{1}{2} e^{-t / \varepsilon} .
$$

Finally the perturbation approximation is given by the sum of the (matched) inner and outer solutions minus the common limiting value around time 0 :

$$
\begin{aligned}
y_{1}(t)+y_{2}(t)-\lim _{\varepsilon \rightarrow 0} y_{1}(u \sqrt{\varepsilon}) & =\left(\frac{t^{2}}{2}+\frac{1}{2}\right)+\left(\frac{1}{2}+\frac{1}{2} e^{-t / \varepsilon}\right)-\frac{1}{2} \\
& =\frac{t^{2}}{2}+\frac{1}{2}+\frac{1}{2} e^{-t / \varepsilon} .
\end{aligned}
$$

\subsection{Howison-Steinberg approximation}

Howison and Steinberg (2005) and Howison (2005) use both inner and outer solutions to get very accurate approximation for discrete barrier options and Bermudan (discrete American) options. Indeed, the approximation not only gives the first order correction as in Broadie et al. (1997), it also leads to a second order correction.

The outer expansion is carried out assuming that the underlying asset price is away from the barrier; in this case a barrier option price can be approximated by the price for the corresponding standard call and put options. The inner solution corresponds to the case when the asset price is close to the barrier.

Since the barrier crossing is only monitored at some discrete time points, the resulting inner solution will be a periodic heat equation. Howison and Steinberg (2005) present an elegant asymptotic analysis of the periodic heat equation by using the result of the Spitzer function (Spitzer, 1957, 1960) for the Wiener-Hopf equation. Afterwards, they matched the inner and outer solutions to get expansions. We shall not give the mathematical details here, and ask the interested reader to read the inspiring papers by Howison and Steinberg (2005) and Howison (2005).

In fact the approximation in Howison and Steinberg (2005) is so good that it can formally give the second order approximation with the order $o(1 / m)$ for discrete barrier options, which is more accurate than the order $o(1 / \sqrt{m})$ in the continuity correction in Broadie et al. (1997). The only drawback seems to be that perturbation methods generally lack rigorous proofs. 


\section{A Laplace transform method via Spitzer's identity}

Building on the result in Ohgren (2001) and the Laplace transform (with respect to log-strike prices) introduced in Carr and Madan (1999), Petrella and Kou (2004) developed a method based on Laplace transform that easily allows us to compute the price and hedging parameters (the Greeks) of discretely monitored lookback and barrier options at any point in time, even if the previous achieved maximum (minimum) cannot be ignored. The method in Petrella and Kou (2004) can be implemented not only under the classical Brownian model, but also under more general models (e.g. jump-diffusion models) with stationary independent increments. A similar method using Fourier transforms in the case of pricing discrete lookback options at the monitoring points (but not at any time points hence with no discussion of the hedging parameters) was independently suggested in Borovkov and Novikov (2002, pp. 893-894). The method proposed in Petrella and Kou (2004) is more general, as it is applicable to price both discrete lookback and barrier options at any time points (hence to compute the hedging parameters).

\subsection{Spitzer's identity and a related recursion}

Consider the asset value $S(t)$, monitored in the interval $[0, T]$ at a sequence of equally spaced monitoring points, $0 \equiv t(0)<t(1)<\cdots<t(m) \equiv T$. Let $X_{i}:=\log \{S(t(i)) / S(t(i-1))\}$, where $X_{i}$ is the return between $t(i-1)$ and $t(i)$. Denote $t(l)$ to be a monitoring point such that time $t$ is between the $(l-1)$ th and $l$ th monitoring points, i.e., $t(l-1) \leqslant t<t(l)$. Define the maxima of the return process between the monitoring points to be $\widetilde{M}_{l, k}:=$ $\max _{l \leqslant j \leqslant k} \sum_{i=l+1}^{j} X_{i}, l=0, \ldots, k$, where we have used the convention that the sum is zero if the index set is empty. Assume that $X_{1}, X_{2}, \ldots$, are independent identically distributed (i.i.d.) random variables. With $X_{s, t}:=\log \{S(t) / S(s)\}$ being the return between time $s$ and time $t, t \geqslant s$, define

$$
C(u, v ; t):=\mathrm{E}^{*}\left[e^{u X_{t, t(l)}}\right] \mathrm{E}^{*}\left[e^{u \widetilde{M}_{l, m}+v X_{t, T}}\right]=\hat{x}_{l, m} \mathrm{E}^{*}\left[e^{(u+v) X_{t, t(l)}}\right],
$$

where

$$
\hat{x}_{l, k}:=\mathrm{E}^{*}\left[e^{u \tilde{M}_{l, k}+v B_{l, k}}\right], \quad l \leqslant k ; \quad B_{l, k}:=\sum_{i=l+1}^{k} X_{i} .
$$

Define for $0 \leqslant l \leqslant k$,

$$
\hat{a}_{l, k}:=\mathrm{E}^{*}\left[e^{(u+v) B_{l, k}^{+}}\right]+\mathrm{E}^{*}\left[e^{-v B_{l, k}^{-}}\right]-1, \quad u, v \in \mathbb{C} .
$$

Spitzer (1956) proved that, for $s<1$ and $u, v \in C$, with $\operatorname{Im}(u) \geqslant 0$ and $\operatorname{Im}(v) \geqslant 0$ :

$$
\sum_{k=0}^{\infty} s^{k} \hat{x}_{l, k}=\exp \left(\sum_{k=1}^{\infty} \frac{s^{k}}{k} \hat{a}_{l, k}\right)
$$


where $B_{l, k}^{+}$and $B_{l, k}^{-}$denote the positive and negative part of the $B_{l, k}$, respectively. We can easily extend (10) to any $u, v \in C$, by limiting $s \leqslant s_{0}^{\prime}$ for some $s_{0}^{\prime}$ small enough.

To get $\hat{x}_{l, k}$ from $\hat{a}_{l, k}$ we can invert the Spitzer's identity by using Leibniz's formula at $s=0$, as in Ohgren (2001). In fact, Petrella and Kou (2004) show that for any given $l$, we have

$$
\hat{x}_{l, k+1}=\frac{1}{k-l+1} \sum_{j=0}^{k-l} \hat{a}_{l, k+1-j} \hat{x}_{l, l+j} .
$$

To compute $\hat{a}_{l, k}$, when $u$ and $v$ are real numbers, Petrella and Kou (2004) also show that

$$
\begin{gathered}
\mathrm{E}^{*}\left[e^{u B_{l, k}^{+}}\right]=\left\{\begin{array}{c}
1+\mathrm{E}^{*}\left[\left(e^{u B_{l, k}}-1\right) \mathbf{1}_{\left\{u B_{l, k}>0\right\}}\right]=1+C_{1}(u, k), \\
\text { if } u \geqslant 0, \\
1-\mathrm{E}^{*}\left[\left(1-e^{u B_{l, k}}\right) \mathbf{1}_{\left\{u B_{l, k}<0\right\}}\right]=1-P_{1}(u, k), \\
\text { if } u<0,
\end{array}\right. \\
\mathrm{E}^{*}\left[e^{-v B_{l, k}^{-}}\right]=\left\{\begin{array}{c}
1+\mathrm{E}^{*}\left[\left(e^{-v B_{l, k}}-1\right) \mathbf{1}_{\left\{v B_{l, k}<0\right\}}\right]=1+C_{1}(-v, k), \\
\text { if } v \geqslant 0, \\
1-\mathrm{E}^{*}\left[\left(1-e^{-v B_{l, k}}\right) \mathbf{1}_{\left\{v B_{l, k}>0\right\}}\right]=1-P_{1}(-v, k), \\
\text { if } v<0,
\end{array}\right.
\end{gathered}
$$

where $C_{1}(u, k)$ is the value of a European call option with strike $K=1$ on the asset $\bar{S}_{t}$ with $\bar{S}_{0}=1$ and return $u \cdot X_{t(l), t(k)}$ (ignoring the discount factor), and $P_{1}(u, k)$ is the value of a European put option with strike $K=1$ on the asset $\bar{S}_{t}$ with $\bar{S}_{0}=1$ and return $u \cdot X_{t(l), t(k)}$. In other words, we can easily compute $\hat{a}_{l, k}$ via analytical solutions of the standard call and put options.

\subsection{Laplace transform for discrete barrier options}

To save the space, we shall only discuss the case of barrier options, as the case of lookback options can be treated similarly; see Petrella and Kou (2004). Let $\xi>1$ and $\zeta>0$ and assume that $C(-\zeta, 1-\xi ; t)<\infty$. At any time $t \in[t(l-1), t(l)), m \geqslant l \geqslant 1$, Petrellla and Kou (2004) show that the double Laplace transform of $f(\kappa, h ; S(t))=\mathrm{E}^{*}\left[\left(e^{\kappa}-S(T)\right)^{+} \mathbf{1}_{\left\{M_{0, T}<e^{h}\right\}} \mid \mathcal{F}_{t}\right]$ is given by

$$
\begin{aligned}
\hat{f}(\xi, \zeta) & :=\int_{-\infty}^{\infty} \int_{-\infty}^{\infty} e^{-\xi \kappa-\zeta h} f(\kappa, h ; S(t)) \mathrm{d} \kappa \mathrm{d} h \\
& =(S(t))^{-(\xi+\zeta-1)} \cdot \frac{C(-\zeta, 1-\xi ; t)}{\xi(\xi-1) \zeta}
\end{aligned}
$$


with the function $C$ defined in (7). The Greeks can also be computed similarly. For example, at any time $t \in[t(l-1), t(l))$, with $1 \leqslant l \leqslant m$, we have:

$$
\begin{aligned}
& \frac{\partial}{\partial S(t)} \operatorname{UOP}(t, T) \\
& =-e^{-r(T-t)} \mathcal{L}_{\xi, \zeta}^{-1}\left(\frac{(\xi+\zeta-1)(S(t))^{-(\xi+\zeta)}}{\xi(\xi-1) \zeta}\right. \\
& \quad \times C(-\zeta, 1-\xi ; t))\left.\right|_{\log (K), \log (H)} \cdot
\end{aligned}
$$

To illustrate the algorithm, without loss of generality, we shall focus on computing the price and the hedging parameters (the Greeks) for an up-and-out put option.

\section{The Algorithm:}

Input: Analytical formulae of standard European call and put options.

Step 1: Use the European call and put formulae to calculate $\hat{a}_{i, k}$, via (9), (12) and (13).

Step 2: Use the recursion in Eq. (11) to compute $\hat{x}_{l, k}$.

Step 3: Compute $C(u, v ; t)$ from Eq. (7).

Step 4: Numerically invert the Laplace transforms given in Eq. (14).

In Step 4 the Laplace transforms are inverted by using two-sided Euler inversion algorithms in Petrella (2004), which are extensions of one-sided Euler algorithms in Abate and Whitt (1992) and Choudhury et al. (1994).

The algorithm essentially only requires to input the standard European call and put prices, thanks to Spitzer's identity. The algorithm can also be extended to price other derivatives, whose values are a function of the joint distribution of the terminal asset value and its discretely monitored maximum (or minimum) throughout the lifetime of the option, such as partial lookback options. As demonstrated in the numerical examples in Petrella and Kou (2004), for a wide variety of parameters (including the cases where the barrier is very close to the initial asset price and there are many monitoring points), the algorithm is quite fast (typically only requires a few seconds), and is quite accurate (typically up to three decimal points). The total workload for both barrier and lookback options is of the order $O\left(N M^{2}\right)$, where $N$ is the number terms needed for Laplace inversion, and $M$ is the total number of monitoring points.

\section{Which method to use}

So far we have introduced four recent methods tailored to discrete barrier and lookback options. A natural question is which method is suitable for your particular needs. The answer really depends on four considerations: speed, accuracy, generality (e.g. whether a method is applicable to models beyond the standard Brownian model), and programming effort. 
The consideration of programming effort is often ignored in the literature, although we think it is important. For example, the popularity of binomial trees and Monte Carlo methods in computational finance is a testimony that simple algorithms with little programming effort are better than faster but more complicated methods. This is also because the CPU time improves every year with increasing computer technology. Therefore, an algorithm not only compete with other algorithms but also with ever faster microprocessors. A tenfold increase in the computation speed of an algorithm is less important five years from now, but the simplicity of the algorithm will remain throughout time.

In terms of speed and programming effort, the fastest and easiest ones are the approximation methods, such as the continuity correction and Howison and Steinberg method, as they have analytical solutions. However, approximations will not yield exact results. More precisely, if you can tolerate about 5 to $10 \%$ pricing error (which is common in practice, as the bid-ask spreads for standard call/put options are in the range of 5 to $10 \%$ and the bid-ask spreads for barrier and lookback options are even more), then you should choose the approximation methods. A drawback for the approximation methods is that it is not clear how to generalize the approximations outside the classical Brownian model.

If accuracy is of great concern, e.g. when you need to set up some numerical benchmarks, then the "exact" methods will be needed. For example, if you use the standard Brownian model or models that only involves normal random variables (such as Merton's normal jump diffusion model), then Broadie-Yamamoto method via the fast Gaussian transform is perhaps the best choice, as it is very fast and accurate, and it is quite easy to implement.

However, if you want to price options under more general Levy processes for a broader class of return processes, including non-Gaussian distributions (e.g. the double exponential jump-diffusion model), which may not be easily written as a mixture of independent Gaussian random variables, then FengLinetsky or the Laplace transform via Spitzer's identity may be appropriate. Feng-Linetsky method is a powerful method that can produce very accurate answers in a fast way, and is faster than the Laplace method via Spitzer's identity; but it perhaps requires more programming effort (related to computing Hilbert transforms) than the Laplace transform method. Furthermore, it is very easy to compute, almost at no additional computational cost, the hedging parameters (the Greeks) using the Laplace transform method via Spitzer's identity.

\section{Appendix A. Proof of (1)}

By considering the events $\left\{\tau^{\prime}(a /(\sigma \sqrt{T}), W)=1\right\}$ and $\left\{\tau^{\prime}(a /(\sigma \sqrt{T})\right.$, $W)=2\}$, we have 


$$
\begin{gathered}
V_{3}(H)=S(0) \sum_{i=1}^{2} \hat{P}\left(W_{3} \geqslant \frac{\log (K / S(0))}{\sigma \sqrt{\Delta t}}, \tau^{\prime}(a /(\sigma \sqrt{T}), W)=i\right) \\
-K e^{-r T} \sum_{i=1}^{2} P^{*}\left(W_{3} \geqslant \frac{\log (K / S(0))}{\sigma \sqrt{\Delta t}}, \tau^{\prime}(a /(\sigma \sqrt{T}), W)=i\right) \\
=S(0) \hat{P}\left(-W_{1} \leqslant-\frac{\log (H / S(0))}{\sigma \sqrt{T}} \sqrt{3},-W_{3} \leqslant-\frac{\log (K / S(0))}{\sigma \sqrt{\Delta t}}\right) \\
+S(0) \hat{P}\left(W_{1}<\frac{\log (H / S(0))}{\sigma \sqrt{T}} \sqrt{3}\right. \\
-W_{2} \leqslant-\frac{\log (H / S(0))}{\sigma \sqrt{T}} \sqrt{3}, \\
\left.-W_{3} \leqslant-\frac{\log (K / S(0))}{\sigma \sqrt{\Delta t}}\right) \\
-K e^{-r T} P^{*}\left(-W_{1} \leqslant-\frac{\log (H / S(0))}{\sigma \sqrt{T}} \sqrt{3}\right. \\
\left.-W_{3} \leqslant-\frac{\log (K / S(0))}{\sigma \sqrt{\Delta t}}\right) \\
-K e^{-r T} P^{*}\left(W_{1}<\frac{\log (H / S(0))}{\sigma \sqrt{T}} \sqrt{3}\right. \\
-W_{2} \leqslant-\frac{\log (H / S(0))}{\sigma \sqrt{T}} \sqrt{3} \\
\left.-W_{3} \leqslant \frac{\log (K / S(0))}{\sigma \sqrt{\Delta t}}\right) .
\end{gathered}
$$

Observe the correlations

$$
\begin{aligned}
& \varrho\left(W_{1}, W_{2}\right)=\varrho\left(Z_{1}, Z_{1}+Z_{2}\right)=\sqrt{1 / 2}=\sqrt{t_{1} / t_{2}}, \\
& \varrho\left(W_{1},-W_{3}\right)=\varrho\left(Z_{1},-Z_{1}-Z_{2}-Z_{3}\right)=-\sqrt{t_{1} / T}, \\
& \varrho\left(W_{2},-W_{3}\right)=\varrho\left(Z_{1}+Z_{2},-Z_{1}-Z_{2}-Z_{3}\right)=-\sqrt{t_{2} / T},
\end{aligned}
$$

and

$$
\begin{aligned}
& \operatorname{Var}\left(W_{k}\right)=k, \quad \hat{\mathrm{E}}\left(W_{k}\right)=k \frac{r+\frac{1}{2} \sigma^{2}}{\sigma} \sqrt{\Delta t}, \\
& \mathrm{E}^{*}\left(W_{k}\right)=k \frac{r-\frac{1}{2} \sigma^{2}}{\sigma} \sqrt{\Delta t}, \quad k=1,2,3 .
\end{aligned}
$$

Note some identities for calculation related to $\hat{P}$

$$
\pm \frac{\log (H / S(0))}{\sigma \sqrt{\Delta t}}-\left\{\left(r+\frac{1}{2} \sigma^{2}\right) / \sigma\right\} \sqrt{\Delta t}
$$




$$
\begin{gathered}
=\frac{ \pm \log (H / S(0))-\left\{\left(r+\frac{1}{2} \sigma^{2}\right)\right\} t_{1}}{\sigma \sqrt{t_{1}}} \equiv \hat{a}_{1, H, \pm}, \\
\frac{1}{\sqrt{2}}\left( \pm \frac{\log (H / S(0))}{\sigma \sqrt{\Delta t}}-2\left\{\left(r+\frac{1}{2} \sigma^{2}\right) / \sigma\right\} \sqrt{\Delta t}\right) \\
=\frac{ \pm \log (H / S(0))-\left\{\left(r+\frac{1}{2} \sigma^{2}\right)\right\} t_{2}}{\sigma \sqrt{t_{2}}} \equiv \hat{a}_{2, H, \pm}, \\
\frac{1}{\sqrt{3}}\left(-\frac{\log (K / S(0))}{\sigma \sqrt{\Delta t}}-3\left\{\left(r+\frac{1}{2} \sigma^{2}\right) / \sigma\right\} \sqrt{\Delta t}\right) \\
=\frac{-\log (K / S(0))-\left\{\left(r+\frac{1}{2} \sigma^{2}\right)\right\} T}{\sigma \sqrt{T}} \equiv \hat{a}_{K},
\end{gathered}
$$

and some identities for calculation related to $P^{*}$

$$
\begin{aligned}
& \pm \frac{\log (H / S(0))}{\sigma \sqrt{\Delta t}}-\left\{\left(r-\frac{1}{2} \sigma^{2}\right) / \sigma\right\} \sqrt{\Delta t} \\
& =\frac{ \pm \log (H / S(0))-\left\{\left(r-\frac{1}{2} \sigma^{2}\right)\right\} t_{1}}{\sigma \sqrt{t_{1}}} \equiv a_{1, H, \pm}^{*}, \\
& \frac{1}{\sqrt{2}}\left( \pm \frac{\log (H / S(0))}{\sigma \sqrt{\Delta t}}-2\left\{\left(r-\frac{1}{2} \sigma^{2}\right) / \sigma\right\} \sqrt{\Delta t}\right) \\
& =\frac{ \pm \log (H / S(0))-\left\{\left(r-\frac{1}{2} \sigma^{2}\right)\right\} t_{2}}{\sigma \sqrt{t_{2}}} \equiv a_{2, H, \pm}^{*}, \\
& \frac{1}{\sqrt{3}}\left(-\frac{\log (K / S(0))}{\sigma \sqrt{\Delta t}}-3\left\{\left(r-\frac{1}{2} \sigma^{2}\right) / \sigma\right\} \sqrt{\Delta t}\right) \\
& =\frac{-\log (K / S(0))-\left\{\left(r-\frac{1}{2} \sigma^{2}\right)\right\} T}{\sigma \sqrt{T}} \equiv a_{K}^{*},
\end{aligned}
$$

from which the conclusion follows.

\section{Appendix B. Calculation of the constant $\beta$}

First of all, we show that the series in (5)

$$
\sum_{n=1}^{\infty}\left\{\frac{1}{\sqrt{n}}-\sqrt{\pi}\left(\begin{array}{c}
-1 / 2 \\
n
\end{array}\right)(-1)^{n}\right\}
$$

converges absolutely. Using Stirling's formula (e.g. Chow and Teicher, 1997)

$$
n !=n^{n} e^{-n} \sqrt{2 \pi n} \cdot \varepsilon_{n}, e^{\frac{1}{12 n+1}}<\varepsilon_{n}<e^{\frac{1}{12 n}},
$$


we have

$$
\begin{aligned}
\sqrt{\pi}\left(\begin{array}{c}
-1 / 2 \\
n
\end{array}\right)(-1)^{n}= & \sqrt{\pi} \frac{1}{2} \frac{3}{2} \cdots \frac{(2 n-1)}{2} / n !=\sqrt{\pi} \frac{(2 n) !}{2^{2 n}} \frac{1}{n ! n !} \\
= & \sqrt{\pi} \frac{(2 n)^{2 n} e^{-2 n} \sqrt{2 \pi \cdot 2 n}}{2^{2 n}} \\
& \times \frac{1}{n^{n} e^{-n} \sqrt{2 \pi n} \cdot n^{n} e^{-n} \sqrt{2 \pi n}} \frac{\varepsilon_{2 n}}{\varepsilon_{n} \varepsilon_{n}} \\
= & \frac{1}{\sqrt{n}} \frac{\varepsilon_{2 n}}{\varepsilon_{n} \varepsilon_{n}} .
\end{aligned}
$$

Since $\frac{\varepsilon_{2 n}}{\varepsilon_{n} \varepsilon_{n}}=1+O(1 / n)$, we have the terms inside the series (15):

$$
\begin{aligned}
\frac{1}{\sqrt{n}}-\sqrt{\pi}\left(\begin{array}{c}
-1 / 2 \\
n
\end{array}\right)(-1)^{n}=\frac{1}{\sqrt{n}}-\frac{1}{\sqrt{n}} \frac{\varepsilon_{2 n}}{\varepsilon_{n} \varepsilon_{n}} & =\frac{1}{\sqrt{n}}\left(1-\frac{\varepsilon_{2 n}}{\varepsilon_{n} \varepsilon_{n}}\right) \\
& =O\left(\frac{1}{n \sqrt{n}}\right),
\end{aligned}
$$

from which we know that the series (15) converges absolutely.

Next, in the case of the standard normal density we have

$$
\beta=\frac{E\left(A_{N}^{2}\right)}{2 E\left(A_{N}\right)}=\frac{\omega_{2}}{\sqrt{2}}=\frac{1}{\sqrt{2}}\left(1-\frac{1}{\sqrt{\pi}} \sum_{n=1}^{\infty}\left\{\frac{1}{\sqrt{n}}-\sqrt{\pi}\left(\begin{array}{c}
-\frac{1}{2} \\
n
\end{array}\right)(-1)^{n}\right\}\right) .
$$

It was shown in Hardy (1905) that

$$
\lim _{x \uparrow 1} \sum_{n=1}^{\infty}\left(\frac{x^{n}}{n^{s}}-\Gamma(1-s)\left\{\log \left(\frac{1}{x}\right)\right\}^{s-1}\right)=\zeta(s) .
$$

Taking $s=1 / 2$ and using the fact that $\Gamma(1 / 2)=\sqrt{\pi}$, we have

$$
\lim _{x \uparrow 1} \sum_{n=1}^{\infty} \frac{x^{n}}{\sqrt{n}}-\sqrt{\pi}\left\{\log \left(\frac{1}{x}\right)\right\}^{-1 / 2}=\zeta(1 / 2) .
$$

Furthermore, letting $x=1-\varepsilon$ yields

$$
\begin{aligned}
& \frac{1}{\sqrt{1-x}}-\left\{\log \left(\frac{1}{x}\right)\right\}^{-1 / 2} \\
& =\frac{\sqrt{\log (1 / x)}-\sqrt{1-x}}{\sqrt{\log (1 / x)} \sqrt{1-x}} \\
& =\frac{\log (1 / x)-(1-x)}{\sqrt{\log (1 / x)} \sqrt{1-x}(\sqrt{\log (1 / x)}+\sqrt{1-x})} \\
& =\frac{-\log (1-\varepsilon)-\varepsilon}{\sqrt{-\log (1-\varepsilon)} \sqrt{\varepsilon}(\sqrt{-\log (1-\varepsilon)}+\sqrt{\varepsilon})}
\end{aligned}
$$




$$
=\frac{O\left(\varepsilon^{2}\right)}{O(\sqrt{\varepsilon}) O(\sqrt{\varepsilon})\{O(\sqrt{\varepsilon})+O(\sqrt{\varepsilon})\}}=O(\sqrt{\varepsilon}) \rightarrow 0,
$$

as $x \uparrow 1$. Therefore, we have

$$
\lim _{x \uparrow 1}\left(\frac{1}{\sqrt{1-x}}-\left\{\log \left(\frac{1}{x}\right)\right\}^{-1 / 2}\right)=0 .
$$

This is interesting, as the both terms $1 / \sqrt{1-x}$ and $\left\{\log \left(\frac{1}{x}\right)\right\}^{-1 / 2}$ go to infinity as $x \uparrow 1$ but the difference goes to zero.

The above limit, in conjunction with (16), yields

$$
\lim _{x \uparrow 1} \sum_{n=1}^{\infty} \frac{x^{n}}{\sqrt{n}}-\sqrt{\pi} \frac{1}{\sqrt{1-x}}=\zeta(1 / 2) .
$$

Since $(1-x)^{-\alpha}=\sum_{n=0}^{\infty}\left(\begin{array}{c}-\alpha \\ n\end{array}\right)(-x)^{n}$, we have

$$
\lim _{x \uparrow 1} \sum_{n=1}^{\infty} \frac{x^{n}}{\sqrt{n}}-\sqrt{\pi} \sum_{n=1}^{\infty}\left(\begin{array}{c}
-1 / 2 \\
n
\end{array}\right)(-x)^{n}-\sqrt{\pi}\left(\begin{array}{c}
-1 / 2 \\
0
\end{array}\right)(-x)^{0}=\zeta(1 / 2) .
$$

In other words,

$$
\lim _{x \uparrow 1} \sum_{n=1}^{\infty}\left\{\frac{1}{\sqrt{n}}-\sqrt{\pi}\left(\begin{array}{c}
-1 / 2 \\
n
\end{array}\right)(-1)^{n}\right\} x^{n}=\sqrt{\pi}+\zeta(1 / 2),
$$

and

$$
\sum_{n=1}^{\infty}\left\{\frac{1}{\sqrt{n}}-\sqrt{\pi}\left(\begin{array}{c}
-1 / 2 \\
n
\end{array}\right)(-1)^{n}\right\}=\sqrt{\pi}+\zeta(1 / 2),
$$

because the series (15) converges absolutely so that we can interchange the limit and summation.

In summary we have

$$
\begin{aligned}
\beta & =\frac{1}{\sqrt{2}}\left(1-\frac{1}{\sqrt{\pi}} \sum_{n=1}^{\infty}\left\{\frac{1}{\sqrt{n}}-\sqrt{\pi}\left(\begin{array}{c}
-\frac{1}{2} \\
n
\end{array}\right)(-1)^{n}\right\}\right) \\
& =\frac{1}{\sqrt{2}}\left(1-\frac{1}{\sqrt{\pi}}\{\sqrt{\pi}+\zeta(1 / 2)\}\right)=-\frac{\zeta(1 / 2)}{\sqrt{2 \pi}} .
\end{aligned}
$$

\section{References}

Abate, J., Whitt, W. (1992). The Fourier series method for inverting transforms of probability distributions. Queueing Systems 10, 5-88.

Ahn, D.G., Figlewski, S., Gao, B. (1999). Pricing discrete barrier options with an adaptive mesh. Journal of Derivatives 6, 33-44. 
Ait-Sahalia, F., Lai, T.L. (1997). Valuation of discrete barrier and hindsight options. Journal of Financial Engineering 6, 169-177.

Ait-Sahalia, F., Lai, T.L. (1998). Random walk duality and valuation of discrete lookback options. Applied Mathematical Finance 5, 227-240.

Andricopoulos, A., Widdicks, M., Duck, P., Newton, D. (2003). Universal option valuation using quadrature methods. Journal of Financial Economics 67, 447-471.

Andreasen, J. (1998). The pricing of discretely sampled Asian and lookback options: A change of numeraire approach. Journal of Computational Finance 2, 5-30.

Asmussen, S., Glynn, P., Pitman, J. (1995). Discretization error in simulation of one-dimensional reflecting Brownian motion. Annals of Applied Probability 5, 875-896.

Babbs, S. (1992). Binomial valuation of lookback options. Working paper. Midland Global Markets, London.

Blanchet, J., Glynn, P. (2006). Complete corrected diffusion approximations for the maximum of random walk. Annals of Applied Probability, in press.

Black, F., Cox, J. (1976). Valuing corporate debts: Some effects of bond indenture provisions. Journal of Finance 31, 351-367.

Borovkov, K., Novikov, A. (2002). On a new approach to calculating expectations for option pricing. Journal of Applied Probability 39, 889-895.

Boyle, P.P., Lau, S.H. (1994). Bumping up against the barrier with the binomial method. Journal of Derivatives 2, 6-14.

Boyle, P.P., Tian, Y. (1998). An explicit finite difference approach to the pricing of barrier options. Applied Mathematical Finance 5, 17-43.

Boyle, P.P., Tian, Y. (1999). Pricing lookback and barrier options under the CEV process. Journal of Financial and Quantitative Analysis 34, 241-264.

Broadie, M., Yamamoto, Y. (2003). A double-exponential fast Gauss transform algorithm for pricing discrete path-dependent options. Working paper. Columbia University. Operations Research (in press).

Broadie, M., Glasserman, P., Kou, S.G. (1997). A continuity correction for discrete barrier options. Mathematical Finance 7, 325-349.

Broadie, M., Glasserman, P., Kou, S.G. (1999). Connecting discrete and continuous path-dependent options. Finance and Stochastics 3, 55-82.

Cao, M., Kou, S.G. (2007). Continuity correction for two dimensional and partial barrier options. Working paper. Columbia University.

Carr, P., Madan, D.B. (1999). Option valuation using the fast Fourier transform. Journal of Computational Finance 2, 61-73.

Chance, D.M. (1994). The pricing and hedging of limited exercise of caps and spreads. Journal of Financial Research 17, 561-584.

Chang, J.T., Peres, Y. (1997). Ladder heights, Gaussian random walks and the Riemann zeta function. Annals of Probability 25, 787-802.

Chen, N., Kou, S.G. (2005). Credit spreads, optimal capital structure, and implied volatility with endogenous default and jump risk. Preprint. Columbia University. Mathematical Finance (in press).

Chernoff, H. (1965). Sequential Tests for the Mean of a Normal Distribution IV. Annals of Mathematical Statistics 36, 55-68.

Cheuk, T., Vorst, T. (1997). Currency lookback options and the observation frequency: A binomial approach. Journal of International Money and Finance 16, 173-187.

Choudhury, G.L., Lucantoni, D.M., Whitt, W. (1994). Multidimensional transform inversion with applications to the transient M/M/1 queue. Annals of Applied Probability 4, 719-740.

Chow, Y.S., Teicher, H. (1997). Probability Theory: Independence, Interchangeability, Martingales, third ed. Springer, New York.

Chuang, C.S. (1996). Joint distributions of Brownian motion and its maximum, with a generalization to corrected BM and applications to barrier options. Statistics and Probability Letters 28, 81-90.

Comtet, A., Majumdar, S.N. (2005). Precise asymptotic for a random walker's maximum. Working paper. Institut Henri Poincaré, Paris, France. 
Conze, R., Viswanathan, R. (1991). Path-dependent options: The case of lookback options. Journal of Finance 46, 1893-1907.

Davydov, D., Linetsky, V. (2001). Pricing and hedging path-dependent options under the CEV process. Management Science 47, 949-965.

Duan, J.C., Dudley, E., Gauthier, G., Simonato, J.G. (2003). Pricing discrete monitored barrier options by a Markov chain. Journal of Derivatives 10, 9-32.

Feng, L., Linetsky, V. (2005). Pricing discretely monitored barrier options and defaultable bonds in Lévy process models: A Hilbert transform approach. Working paper. Northwestern University. Mathematical Finance (in press).

Figlewski, S., Gao, B. (1999). The adaptive mesh model: A new approach to efficient option pricing. Journal of Financial Economics 53, 313-351.

Fusai, G., Recchioni, M.C. (2003). Analysis of quadrature methods for the valuation of discrete barrier options. Working paper. Universita del Piemonte.

Gatto, M., Goldman, M.B., Sosin, H. (1979). Path-dependent options: "buy at the low, sell at the high". Journal of Finance 34, 1111-1127.

Glasserman, P. (2004). Monte Carlo Methods in Financial Engineering. Springer, New York.

Goldman, M.B., Sosin, H., Shepp, L. (1979). On contingent claims that insure ex post optimal stock market timing. Journal of Finance 34, 401-414.

Hardy, G.H. (1905). A method for determining the behavior of certain classes of power series near a singular point on the circle of convergence. Proceedings London Mathematical Society 2, 381-389.

Haug, E.G. (1999). Barrier put-call transformations. Working paper. Tempus Financial Engineering.

Heynen, R.C., Kat, H.M. (1994a). Crossing barriers. Risk 7 (June), 46-49. Correction (1995), Risk 8 (March), 18. Reprinted in: Jarrow, R. (Ed.), Over the Rainbow: Developments in Exotic Options and Complex Swaps. RISK/FCMC, London, pp. 179-182.

Heynen, R.C., Kat, H.M. (1994b). Partial barrier options. Journal of Financial Engineering 3, 253-274.

Heynen, R.C., Kat, H.M. (1995). Lookback options with discrete and partial monitoring of the underlying price. Applied Mathematical Finance 2, 273-284.

Hörfelt, P. (2003). Extension of the corrected barrier approximation by Broadie, Glasserman, and Kou. Finance and Stochastics 7, 231-243.

Hogan, M. (1986). Comment on a problem of Chernoff and Petkau. Annals of Probability 14, 1058-1063.

Howison, S. (2005). A matched asymptotic expansions approach to continuity corrections for discretely sampled options. Part 2: Bermudan options. Preprint. Oxford University.

Howison, S., Steinberg, M. (2005). A matched asymptotic expansions approach to continuity corrections for discretely sampled options. Part 1: Barrier options. Preprint. Oxford University.

Hull, J.C. (2005). Options, Futures, and Other Derivative Securities, fourth ed. Prentice Hall, Englewood Cliffs, NJ.

Hull, J., White, A. (1993). Efficient procedures for valuing European and American path-dependent options. Journal of Derivatives 1, 21-31.

Karatzas, I., Shreve, S. (1991). Brownian Motion and Stochastic Calculus, second ed. Springer, New York.

Kat, H. (1995). Pricing lookback options using binomial trees: an evaluation. Journal of Financial Engineering 4, 375-397.

Kou, S.G. (2003). On pricing of discrete barrier options. Statistica Sinica 13, 955-964.

Kou, S.G., Wang, H. (2003). First passage times of a jump diffusion process. Advances in Applied Probability $35,504-531$.

Kou, S.G., Wang, H. (2004). Option pricing under a double exponential jump diffusion model. Management Science 50, 1178-1192.

Kuan, G., Webber, N.J. (2003). Valuing discrete barrier options on a Dirichlet lattice. Working paper. University of Exester, UK.

Kunitomo, N., Ikeda, M. (1992). Pricing Options with Curved Boundaries. Mathematical Finance 2, $275-298$.

Lai, T.L. (1976). Asymptotic moments of random walks with applications to ladder variables and renewal theory. Annals of Probability 11, 701-719. 
Leland, H.E., Toft, K. (1996). Optimal capital structure, endogenous bankruptcy, and the term structure of credit spreads. Journal of Finance 51, 987-1019.

Longstaff, F.A. (1995). How much can marketability affect security values? Journal of Finance 50, 17671774.

Merton, R.C. (1973). Theory of rational option pricing. Bell Journal of Economic Management Science 4, 141-183.

Merton, R.C. (1974). On the pricing of corporate debts: the risky structure of interest rates. Journal of Finance 29, 449-469.

Merton, R.C. (1976). Option pricing when underlying stock returns are discontinuous. Journal of Financial Economics 3, 125-144.

Ohgren, A. (2001). A remark on the pricing of discrete lookback options. Journal of Computational Finance 4, 141-147.

Petrella, G. (2004). An extension of the Euler method for Laplace transform inversion algorithm with applications in option pricing. Operations Research Letters 32, 380-389.

Petrella, G., Kou, S.G. (2004). Numerical pricing of discrete barrier and lookback options via Laplace transforms. Journal of Computational Finance 8, 1-37.

Reiner, E. (2000). Convolution methods for path-dependent options. Preprint. UBS Warburg Dillon Read.

Rich, D. (1996). The mathematical foundations of barrier option pricing theory. Advances in Futures Options Research 7, 267-312.

Ritchken, P. (1995). On pricing barrier options. Journal of Derivatives 3, 19-28.

Rubinstein, M., Reiner, E. (1991). Breaking down the barriers. Risk 4 (September), 28-35.

Schroder, M. (1999). Changes of numeraire for pricing futures, forwards and options. Review of Financial Studies 12, 1143-1163.

Siegmund, D. (1979). Corrected diffusion approximation in certain random walk problems. Advances in Applied Probability 11, 701-719.

Siegmund, D. (1985). Sequential Analysis: Tests and Confidence Intervals. Springer-Verlag, New York.

Siegmund, D., Yuh, Y.-S. (1982). Brownian approximations for first passage probabilities. Zeitschrift für Wahrsch. verw. Gebiete 59, 239-248.

Spitzer, F. (1956). A combinatorial lemma and its application to probability theory. Transactions of the American Mathematical Society 82, 323-339.

Spitzer, F. (1957). The Wiener-Hopf equation whose kernel is a probability density. Duke Mathematical Journal 24, 327-343.

Spitzer, F. (1960). The Wiener-Hopf equation whose kernel is a probability density (II). Duke Mathematical Journal 27, 363-372.

Sullivan, M.A. (2000). Pricing discretely monitored barrier options. Journal of Computational Finance 3, 35-52.

Tian, Y. (1999). Pricing complex barrier options under general diffusion processes. Journal of Derivatives 6 (Fall), 51-73.

Tse, W.M., Li, L.K., Ng, K.W. (2001). Pricing discrete barrier and hindsight options with the tridiagonal probability algorithm. Management Science 47, 383-393.

Woodroofe, M. (1982). Nonlinear Renewal Theory in Sequential Analysis. Society for Industrial and Applied Mathematics, Philadelphia.

Zhang, P.G. (1998). Exotic Options, second ed. World Scientific, Singapore.

Zvan, R., Vetzal, K.R., Forsyth, P.A. (2000). PDE methods for pricing barrier options. Journal of Economic Dynamics and Control 24, 1563-1590. 